\title{
Uma Análise de Desempenho sobre uma MicroFog
}

\author{
Douglas Siqueira*, Paulo H. Rocha*, Tomaz Bisneto*, Paulo A. L. Rego ${ }^{\mp}$ e \\ Francisco A. Silva* \\ ${ }^{1} *$ Núcleo de Pesquisas Avançadas em Sistemas Distribuídos (NUPASD) \\ Universidade Federal do Piauí (UFPI) \\ 干Grupo de Redes de Computadores, Engenharia de Software e Sistemas (GREat) \\ Universidade Federal do Ceará (UFC) \\ fapseufpi.edu.br, pauloalraufc.br
}

\begin{abstract}
Resumo. A Computação em Nuvem é um paradigma de distribuição de recursos de processamento e armazenamento pela Internet. Uma área relacionada é a Fog Computing, que é uma Cloud com a proximidade ao usuário final. Pelo fato de que servidores tradicionais adotados na Computação em Nuvem possuem elevado preço e consumo energético. Este trabalho apresenta uma avaliação de desempenho de uma MicroFog (uma Fog de pequeno porte), formada por microcontroladores Raspberry P $i$, comparada com servidores tradicionais. Foi utilizada um algoritmo de processamento de imagens e realizada uma análise de sensibilidade dos fatores: (A) servidores (tradicionais e MicroFog), (B) carga de trabalho e $(C)$ tamanho da imagem.
\end{abstract}

\section{Introdução}

A Computação em Nuvem é um paradigma voltado a resolver problemas de armazenamento e processamento de dados computacionais [Mansur et al. 2010]. As aplicações baseadas no conceito de Nuvem, descentralizam o processamento de um único computador, assim necessitando de servidores, na maioria das vezes bastante potentes. O que gera uma grande parte dos investimentos dentro da arquitetura da nuvem.

Os altos preços de tais equipamentos dificultam a sua aquisição por empresas de pequeno porte [Wilcox et al. 2015]. A busca por redução de custos é um dos itens dominantes no projeto das infraestruturas dos serviços em nuvem [Verdi et al. 2010]. Outro problema em decorrência do uso dos servidores é o seu alto gasto de energia. De acordo com [Shehabi et al. 2016] em 2014, os data centers nos EUA consumiram cerca de 70 bilhões de Killowats, representando cerca de 1,8\% do consumo total de energia dos EUA.

Fog Computing é um paradigma que estende a Cloud e seus serviço, levando-os para a borda da rede [Patil 2015]. O que evita o alto gasto energético. Por esta razão, o presente trabalho desenvolve uma MicroFog, com microcontroladores Raspberry e o compara com outros servidores. Além de utilizar o método Design of Experiments e as métricas consumo de energia, CPU e tempo de resposta para analisar os resultados.

O restante deste trabalho esta estruturado da seguinte forma: A Seção 2 mostra como foram conduzidos os experimentos e seus respectivos resultados. Na Seção 3, são detalhados os trabalhos relacionados. E por fim, a Seção 4 apresenta as conclusões e trabalhos futuros. 


\section{Análise de Desempenho}

Esta seção apresenta o ambiente de desenvolvimento e da análise comparativa, como também dos resultados obtidos com o presente trabalho.

\subsection{Ambiente e Metodologia de Avaliação}

Para este trabalho foi adotado um Broker de Mensagens (o RabbitMQ) utilizado para o envio das imagens. As imagens foram segmentadas usando a biblioteca OpenCV. O envio e a segmentação das imagens foram realizados 48 vezes para cada grupo de imagens com mesmo tamanho. Na Tabela 1 detalha os fatores e níveis escolhidos para a avaliação de desempenho do trabalho.

Tabela 1. Fatores e Níveis da Avaliação de Desempenho

\begin{tabular}{cc}
\hline Fatores & Níveis \\
\hline \multirow{2}{*}{ Servidores } & Servidor 01 (Core i3 4 Núcleos 2,5 Ghz e 7,7 GB de memória RAM) \\
& Servidor 02 (Core i3 4 Núcleos 1,7 Ghz e 4,0 GB de memória RAM \\
& MicroFog de Raspberries \\
\hline \multirow{2}{*}{ Tamanho da Imagem } & Imagem 110 KB \\
& Imagem 320 KB \\
\hline \multirow{2}{*}{ Carga } & 15 Imagens \\
& 30 Imagens \\
\hline
\end{tabular}

Durante os experimentos três métricas foram observadas: consumo de CPU, tempo em segundos, e o gasto de energia em Kilowatt e o preço coletado em diferentes sites de e-commerce no Brasil. A MicroFog proposta neste trabalho possui três Raspberries. Com estes é coletado o tempo de processamento e gasto de CPU por instrumentação de código e o gasto energético é mensurado por um equipamento chamado Wanf WF-D02A ${ }^{1}$.

\subsection{Resultados}

Esta seção apresenta resultados e discussões sobre os experimentos.

\subsubsection{Análise Focando na Carga de Trabalho}

A Figura 1 apresenta um conjunto de gráficos variando as métricas de interesse (CPU, energia e tempo de processamento). Nos resultados obtidos através do consumo de energia (Figuras 1(a) e 1(b)), a MicroFog possui menores valores já o Servidor 02 e Servidor 01 obteve um pequeno aumento.

As Figuras 1(c) e 1(d) nestes gráficos ocorreu o melhor desempenho da MicroFog com relação aos demais equipamentos. Nas Figuras 1(e) e 1(f) exibem os resultados do tempo de processamento. Há um destaque maior no Servidor 01, que obteve um menor tempo por ter o melhor poder computacional.

As Figuras 1(e) e 1(f) exibem os resultados do tempo de processamento. A MicroFog, devido ao seu hardware limitado, leva um tempo maior para processar as imagens. Esta mesma explicação é aplicada ao Servidor 02. Já o Servidor 01, por possuir um poder computacional mais elevado, acaba tendo grande vantagem nesse critério.

A Tabela 2 que mostra o preço médio dos diferentes equipamentos coletados em diferentes sites de e-commerce populares no Brasil. A partir da média obtida desta análise,

\footnotetext{
${ }^{1}$ http://solarlab.se/solpanel/datablad/WF-D02A-energy-meter.pdf
} 


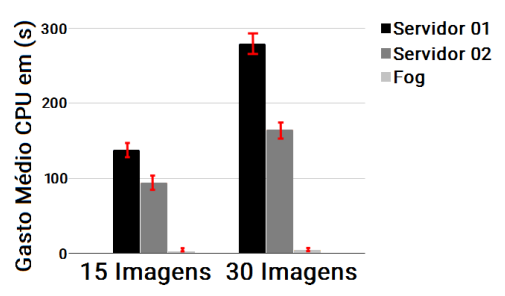

(a) Consumo da CPU com Imagem de $110 \mathrm{kB}$

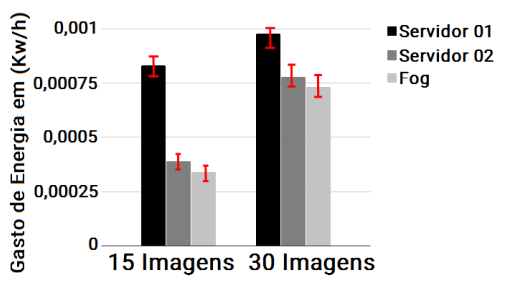

(c) Consumo de Energia com Imagem de $110 \mathrm{kB}$

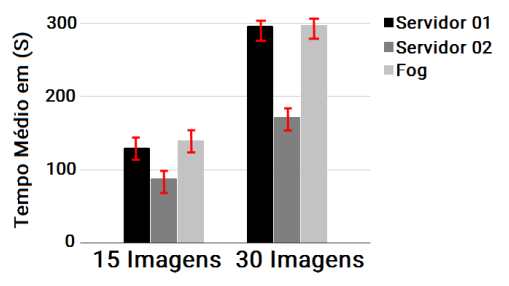

(e) Tempo de Processamento em Segundos com Imagem 110kB

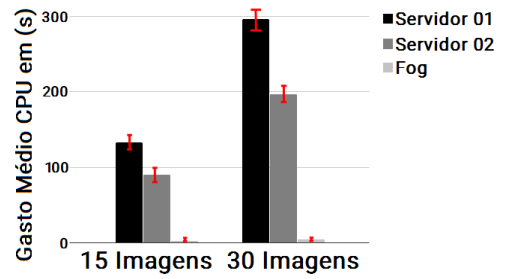

(b) Consumo da CPU com Imagem de $320 \mathrm{kB}$

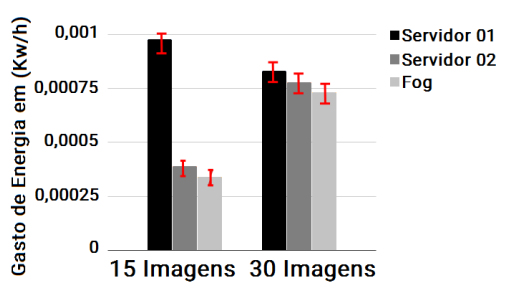

(d) Consumo de Energia com Imagem de $320 \mathrm{kB}$

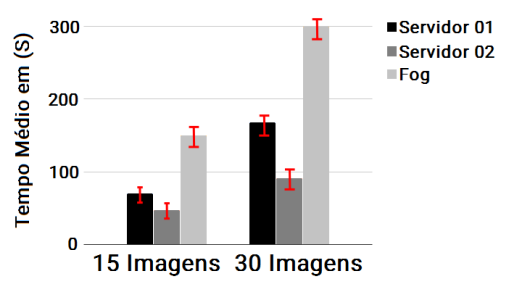

(f) Tempo de Processamento em Segundos com Imagem 320kB

Figura 1. Comparação das Arquiteturas variando a Carga de Trabalho

pode ser determinado que a MicroFog de Raspberries é o equipamento mais viável com relação ao baixo custo.

Tabela 2. Preço dos Equipamentos.

\begin{tabular}{c|c}
\hline Dispositivos & Preço R\$ \\
\hline Servidor 01 & 1849.50 \\
Servidor 02 & 1924.50 \\
MicroFog & 656.25 \\
\hline
\end{tabular}

\subsubsection{Análise Focando nos Fatores}

Nas Figura 2, 3 e 4 apresentam um Design of Experiments (DoE) trata-se de uma técnica de planejamento e análise de experimentos. Nas Tabelas 4, 5 e 6 mostra os valores de efeito obtidos com o DoE. A Figura 2 mostra os efeitos dos fatores sobre o tempo de processamento das imagens pela CPU. Assim como a Tabela 4. O principal efeito desta foi o Tamanho da Imagem. A Figura 3 e a Tabela 5 ilustra os efeitos dos fatores sobre o tempo de execução das imagens. Apesar de apenas dobrar o número de imagens isso não causou um grande efeito. Tendo como o principal a carga neste caso Enquanto a Figura 4 e a Tabela 6 apresentam os efeitos dos fatores sobre a Energia em Kilowatts. Neste caso, 
o fator de maior impacto foi o Tamanho da Imagem. Portanto, deve-se atentar-se se a imagem é grande ou pequena.

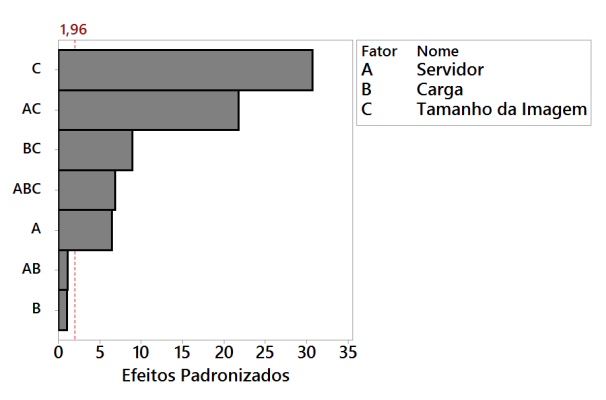

\begin{tabular}{cc}
\hline Fatores & Efeitos \\
\hline $\mathrm{A}$ & $-46,86$ \\
\hline $\mathrm{B}$ & $-0,693$ \\
\hline $\mathrm{C}$ & 2,0140 \\
\hline $\mathrm{A}+\mathrm{B}$ & 0,352 \\
\hline $\mathrm{A}+\mathrm{C}$ & $-0,6605$ \\
\hline $\mathrm{C}+\mathrm{B}$ & 0,02464 \\
\hline $\mathrm{A}+\mathrm{B}+\mathrm{C}$ & $-0,008749$
\end{tabular}

Figura 2. e Tabela 4. Efeitos em Relação a CPU

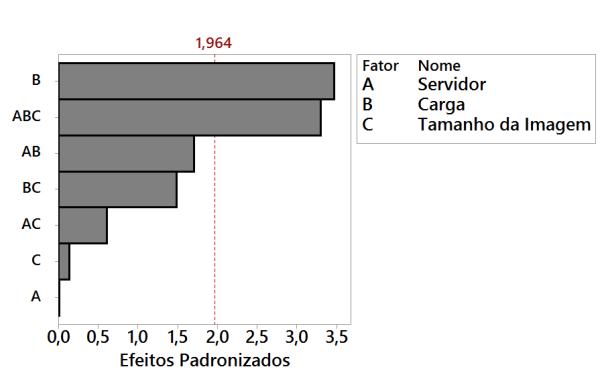

\begin{tabular}{cc}
\hline Fatores & Efeitos \\
\hline $\mathrm{A}$ & 0,4 \\
\hline $\mathrm{B}$ & 19,71 \\
\hline $\mathrm{C}$ & $-0,074$ \\
\hline $\mathrm{A}+\mathrm{B}$ & $-4,48$ \\
\hline $\mathrm{A}+\mathrm{C}$ & 0,157 \\
\hline $\mathrm{C}+\mathrm{B}$ & $-0,0353$ \\
\hline $\mathrm{A}+\mathrm{B}+\mathrm{C}$ & 0,03621 \\
\hline
\end{tabular}

Figura 3. e Tabela 5. Efeitos em Relação ao Tempo de Execução

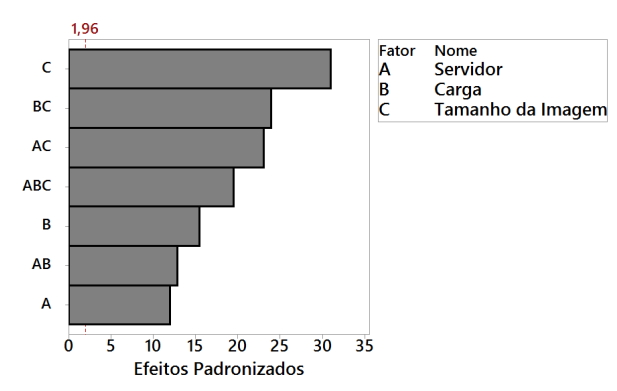

\begin{tabular}{cc}
\hline Fatores & Efeitos \\
\hline $\mathrm{A}$ & 17,37 \\
\hline $\mathrm{B}$ & 21,06 \\
\hline $\mathrm{C}$ & 37,59 \\
\hline $\mathrm{A}+\mathrm{B}$ & 27,18 \\
\hline $\mathrm{A}+\mathrm{C}$ & 17,45 \\
\hline $\mathrm{C}+\mathrm{B}$ & 27,54 \\
\hline $\mathrm{A}+\mathrm{B}+\mathrm{C}$ & 22,68 \\
\hline
\end{tabular}

Figura 4. e Tabela 6. Efeitos em Relação a Energia em Kilowatts

\subsubsection{Análise Focando nos Níveis}

A Figura 5 apresenta gráficos de interação. Nas quais são identificadas por linhas não paralelas. Quanto maior for a inclinação entre as linhas, maior o grau de interação. Linhas paralelas indicam ausência de interação entre os fatores.

O gráfico da Figura 5(a) apresenta a maior interação em termos de Gasto de CPU entre os fatores Carga e Servidor. Assim quando a MicroFog é adotada o impacto da Carga é mais notado. Na Figura 5(b) as três combinações possuem uma certa interação. Visualmente, a inclinação maior é combinando Tamanho da Imagem com Servidor. A Figura 5(c) mostra a interação dos fatores para Energia em Kilowatts. Neste caso, os valores estão condizentes com as tabelas de efeitos apresentadas anteriormente. 


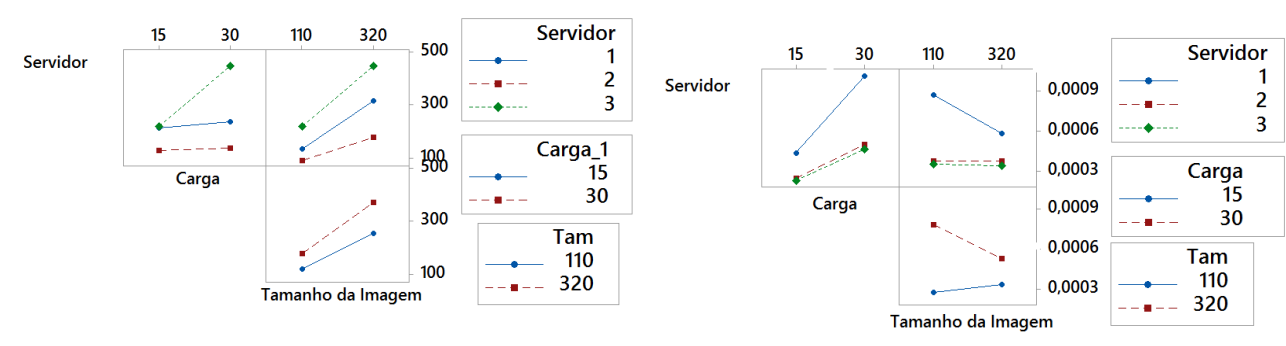

(a) Interação CPU

(b) Interação Tempo de Execução

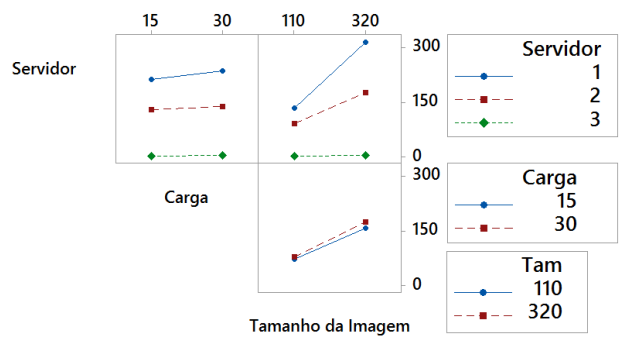

(c) Interação Energia em kilowatts

Figura 5. Interação dos Níveis dos Fatores

\section{Trabalhos Relacionados}

Esta seção apresenta um levantamento sobre os principais trabalhos que criaram Clouds, Fogs ou Clusters com microcontroladores Raspberry Pi ou outros tipos de microcontroladores. No trabalho [Kiepert 2013] foi desenvolvido um cluster com 32 Raspberries. Dentre eles, estavam simuladores de Raspberries para aumentar o poder computacional do cluster. No trabalho [Abrahamsson et al. 2013] foi realizada uma cloud com Raspberries. Como também foi desenvolvido um sistema para controle de cada nó Raspberry.

[Tso et al. 2013] apresenta a Glasgow Raspberry Pi Cloud (PiCloud), um modelo em escala de um Data Center composto de Raspberries. Em [Cloutier et al. 2014] desenvolveu uma cloud com 8 Raspberries e os comparou com outros equipamentos. Dentre eles, placas de desktops, utilizando como métrica principal a CPU dos equipamentos. No trabalho de [Franks and Yerby 2014] há um detalhamento dos desafios da criação de um supercomputador e possibilidades de baixo custo para que a criação seja usada para a indústria.

No artigo de [Princy and Nigel 2015] ocorreu a implementação de uma Cloud de armazenamento de arquivos em tempo real. Isto foi possível utilizando uma aplicação chamada OwnCloud ${ }^{2}$. Em [Jalali et al. 2016] percebeu-se que aplicar uma cloud com Raspbeeries é eficiente em se tratando de aplicações dentro da Internet das Coisas.

\section{Conclusão}

Com o crescente uso das tecnologias em Nuvem, a necessidade de hardware e tecnologias relacionadas está sempre aumentando. A realidade financeira é um grande empecilho que torna a aquisição de equipamentos algo custoso. Assim, este trabalho teve como objetivo desenvolver uma MicroFog com microcontroladores Raspberry Pi e analisar o seu desempenho comparando-a com servidores de pequeno porte. Como resultado a MicroFog gasta

\footnotetext{
${ }^{2}$ https://owncloud.org
} 
menos energia. Na análise de desempenho permitiu analisar 3 fatores: (A) servidores (tradicionais e MicroFog), (B) carga de trabalho e (C) tamanho da imagem. Os resultados apontam que a escolha do servidor teve maior impacto tanto no tempo de execução quanto no gasto de energia. Como trabalho futuro, pretende-se realizar uma análise com outros fatores, como níveis de usuários e requisições simultâneas.

\section{Referências}

Abrahamsson, P., Helmer, S., Phaphoom, N., Nicolodi, L., Preda, N., Miori, L., Angriman, M., Rikkila, J., Wang, X., Hamily, K., et al. (2013). Affordable and energyefficient cloud computing clusters: the bolzano raspberry pi cloud cluster experiment. In Cloud Computing Technology and Science (CloudCom), 2013 IEEE 5th International Conference on, volume 2, pages 170-175. IEEE.

Cloutier, M. F., Paradis, C., and Weaver, V. M. (2014). Design and analysis of a 32bit embedded high-performance cluster optimized for energy and performance. In Proceedings of the 1st International Workshop on Hardware-Software Co-Design for High Performance Computing, pages 1-8. IEEE Press.

Franks, S. and Yerby, J. (2014). Creating a low-cost supercomputer with raspberry pi. In Proceedings of the Southern Association for Information Systems Conference.

Jalali, F., Hinton, K., Ayre, R., Alpcan, T., and Tucker, R. S. (2016). Fog computing may help to save energy in cloud computing. IEEE Journal on Selected Areas in Communications, 34(5):1728-1739.

Kiepert, J. (2013). Creating a raspberry pi-based beowulf cluster. Boise State University, pages $1-17$.

Mansur, A. F. U., Gomes, S. S., Lopes, A. d. A., and Biazus, M. (2010). Novos rumos para a informática na educação pelo uso da computação em nuvem (cloud education): Um estudo de caso do google apps. In Foz do Iguaçu: Anais do XVI Congresso Internacional ABED de Educação a Distância.

Patil, P. V. (2015). Fog computing. College of Engg \& Tech.

Princy, S. E. and Nigel, K. G. J. (2015). Implementation of cloud server for real time data storage using raspberry pi. In Green Engineering and Technologies (IC-GET), 2015 Online International Conference on, pages 1-4. IEEE.

Shehabi, A., Smith, S., Sartor, D., Brown, R., Herrlin, M., Koomey, J., Masanet, E., Horner, N., Azevedo, I., and Lintner, W. (2016). United states data center energy usage report.

Tso, F. P., White, D. R., Jouet, S., Singer, J., and Pezaros, D. P. (2013). The glasgow raspberry pi cloud: A scale model for cloud computing infrastructures. In Distributed Computing Systems Workshops (ICDCSW), 2013 IEEE 33rd International Conference on, pages 108-112. IEEE.

Verdi, F. L., Rothenberg, C. E., Pasquini, R., and Magalhaes, M. (2010). Novas arquiteturas de data center para cloud computing. Minicursos do XXVIII SBRC, pages 103-152.

Wilcox, E., Jhunjhunwala, P., Gopavaram, K., and Herrera, J. (2015). Pi-crust: a raspberry pi cluster implementation. Technical report, Technical report, Texas A\&M University. 